\title{
Measurement of Final-State Correlations in Neutrino Muon-Proton Mesonless Production on Hydrocarbon at $\left\langle E_{\nu}\right\rangle=3 \mathrm{GeV}$
}

X.-G. Lu, ${ }^{1}$ M. Betancourt, ${ }^{2}$ T. Walton, ${ }^{2}$ F. Akbar, ${ }^{3}$ L. Aliaga, ${ }^{4,5}$ O. Altinok, ${ }^{6}$ D. A. Andrade, ${ }^{7}$ M. Ascencio,,${ }^{5}$ L. Bellantoni,${ }^{2}$ A. Bercellie, ${ }^{8}$ A. Bodek, ${ }^{8}$ A. Bravar, ${ }^{9}$ H. Budd,${ }^{8}$ T. Cai, ${ }^{8}$ M. F. Carneiro, ${ }^{10}$ J. Chaves,${ }^{11}$ D. Coplowe, ${ }^{1}$ H. da Motta, ${ }^{12}$ S. A. Dytman, ${ }^{13}$ G. A. Díaz, ${ }^{8,5}$ J. Felix, ${ }^{7}$ L. Fields, ${ }^{2,14}$ R. Fine, ${ }^{8}$ A. M. Gago, ${ }^{5}$ R. Galindo, ${ }^{15}$ H. Gallagher, ${ }^{6}$ A. Ghosh,${ }^{15,12}$ R. Gran, ${ }^{16}$ D. A. Harris, ${ }^{2}$ S. Henry, ${ }^{8}$ S. Jena, ${ }^{17}$ D. Jena, ${ }^{2}$ J. Kleykamp, ${ }^{8}$ M. Kordosky, ${ }^{4}$ T. Le, ${ }^{6,18}$ E. Maher, ${ }^{19}$ S. Manly, ${ }^{8}$ W. A. Mann, ${ }^{6}$ C. M. Marshall, ${ }^{8, *}$ K. S. McFarland, ${ }^{8,2}$ A. M. McGowan, ${ }^{8}$ B. Messerly, ${ }^{13}$ J. Miller, ${ }^{15}$ A. Mislivec, ${ }^{8}$ J. G. Morfín, ${ }^{2}$ J. Mousseau, ${ }^{20, \dagger}$ D. Naples,${ }^{13}$ J. K. Nelson, ${ }^{4}$ C. Nguyen,${ }^{20}$ A. Norrick, ${ }^{4}$ Nuruzzaman, ${ }^{18,15}$ A. Olivier, ${ }^{8}$ V. Paolone, ${ }^{13}$ C. E. Patrick, ${ }^{14,}$ G. N. Perdue,${ }^{2,8}$ M. A. Ramírez, ${ }^{7}$ R. D. Ransome, ${ }^{18}$ L. Ren, ${ }^{13}$ D. Rimal, ${ }^{20}$ P. A. Rodrigues,${ }^{21,8}$ D. Ruterbories, ${ }^{8}$ H. Schellman, ${ }^{10,14}$ J. T. Sobczyk,${ }^{22}$ C. J. Solano Salinas ${ }^{23}$ H. Su, ${ }^{13}$ M. Sultana, ${ }^{8}$ E. Valencia,${ }^{4,7}$ D. Wark, ${ }^{1}$ A. Weber, ${ }^{1}$ J. Wolcott,${ }^{8,}$ M. Wospakrik, ${ }^{20}$ and B. Yaeggy ${ }^{15}$

(MINERvA Collaboration)

\author{
${ }^{1}$ Oxford University, Department of Physics, Oxford, United Kingdom \\ ${ }^{2}$ Fermi National Accelerator Laboratory, Batavia, Illinois 60510, USA \\ ${ }^{3}$ AMU Campus, Aligarh, Uttar Pradesh 202001, India \\ ${ }^{4}$ Department of Physics, College of William \& Mary, Williamsburg, Virginia 23187, USA \\ ${ }^{5}$ Sección Física, Departamento de Ciencias, Pontificia Universidad Católica del Perú, Apartado 1761, Lima, Perú \\ ${ }^{6}$ Physics Department, Tufts University, Medford, Massachusetts 02155, USA \\ ${ }^{7}$ Campus León y Campus Guanajuato, Universidad de Guanajuato, \\ Lascurain de Retana No. 5, Colonia Centro, Guanajuato 36000, Guanajuato México \\ ${ }^{8}$ University of Rochester, Rochester, New York 14627, USA \\ ${ }^{9}$ University of Geneva, 1211 Geneva 4, Switzerland \\ ${ }^{10}$ Department of Physics, Oregon State University, Corvallis, Oregon 97331, USA \\ ${ }^{11}$ Department of Physics and Astronomy, University of Pennsylvania, Philadelphia, Pennsylvania 19104, USA \\ ${ }^{12}$ Centro Brasileiro de Pesquisas Físicas, Rua Dr. Xavier Sigaud 150, Urca, Rio de Janeiro, Rio de Janeiro, 22290-180, Brazil \\ ${ }^{13}$ Department of Physics and Astronomy, University of Pittsburgh, Pittsburgh, Pennsylvania 15260, USA \\ ${ }^{14}$ Northwestern University, Evanston, Illinois 60208, USA \\ ${ }^{15}$ Departamento de Física, Universidad Técnica Federico Santa María, Avenida España 1680 Casilla 110-V, Valparaíso, Chile \\ ${ }^{16}$ Department of Physics, University of Minnesota-Duluth, Duluth, Minnesota 55812, USA \\ ${ }^{17}$ Indian Institute of Science Education and Research, Knowledge city, Sector 81, SAS Nagar, Manauli PO 140306, Mohali, India \\ ${ }^{18}$ Rutgers, The State University of New Jersey, Piscataway, New Jersey 08854, USA \\ ${ }^{19}$ Massachusetts College of Liberal Arts, 375 Church Street, North Adams, Massachusetts 01247, USA \\ ${ }^{20}$ University of Florida, Department of Physics, Gainesville, Florida 32611, USA \\ ${ }^{21}$ University of Mississippi, Oxford, Mississippi 38677, USA \\ ${ }^{22}$ University of Wroclaw, plac Uniwersytecki 1, 50-137 Wrocaw, Poland \\ ${ }^{23}$ Universidad Nacional de Ingeniería, Apartado 31139, Lima, Perú
}

(Received 15 May 2018; revised manuscript received 15 June 2018; published 11 July 2018)

Final-state kinematic imbalances are measured in mesonless production of $\nu_{\mu}+A \rightarrow \mu^{-}+p+X$ in the MINERvA tracker. Initial- and final-state nuclear effects are probed using the direction of the $\mu^{-}-p$ transverse momentum imbalance and the initial-state momentum of the struck neutron. Differential cross sections are compared to predictions based on current approaches to medium modeling. These models underpredict the cross section at intermediate intranuclear momentum transfers that generally exceed the Fermi momenta. As neutrino interaction models need to correctly incorporate the effect of the nucleus in order to predict neutrino energy resolution in oscillation experiments, this result points to a region of phase

Published by the American Physical Society under the terms of the Creative Commons Attribution 4.0 International license. Further distribution of this work must maintain attribution to the author(s) and the published article's title, journal citation, and DOI. Funded by $\operatorname{SCOAP}$. 
space where additional cross section strength is needed in current models, and demonstrates a new technique that would be suitable for use in fine-grained liquid argon detectors where the effect of the nucleus may be even larger.

DOI: 10.1103/PhysRevLett.121.022504

An accurate understanding of nuclear medium modifications to neutrino-nucleon interactions is required for reliable measurements of fundamental neutrino properties. The distributions of final-state observables reflect complicated and intertwined effects from nucleon and nuclear dynamics, and the interpretation of single-particle kinematics is thereby obscured [1]. These underlying dynamics can influence neutrino energy reconstruction in oscillation experiments $[2,3]$. Certain categories of nuclear effects, however, can be separated by variables $[4,5]$ designed to elicit final-state correlations that are absent for neutrino interactions on free nucleons, but are in-play in neutrinonucleus scattering. This Letter reports the measurements with such variables for the purpose of constraining nuclear effects in neutrino interactions.

In charged-current neutrino-nucleus scattering there is an imbalance, $\delta \vec{p}$, between the initial neutrino momentum and the sum of final-state lepton and hadron momenta as a result of nuclear effects. This imbalance is the sum of Fermi motion (FM) and intranuclear momentum transfer (IMT), which is the sum of all other effects including nucleon correlations $[1,6-16]$ and final-state interactions (FSI):

$$
\delta \vec{p}=\vec{p}_{\mathrm{FM}}-\vec{p}_{\mathrm{IMT}} .
$$

In $\nu_{\mu}$ charged-current quasielastic (QE) interactions, $\nu_{\mu}+n \rightarrow \mu^{-}+p$, momentum is transferred from the leptonic current to the target neutron. However, if the neutron is correlated with other nucleons, the momentum transfer is shared among the correlated partners. In both cases, finalstate interactions occur as particles from the primary interaction propagate through the nucleus exchanging energy, momentum, and charge with the nuclear environment. Primary particles can be absorbed during this propagation; baryonic resonances (RES) can be produced in the primary interaction and the resulting products can undergo FSI and be absorbed. These non-QE processes give the same observable final state as QE scattering.

This study focuses on the QE-like process

$$
\nu_{\mu}+A \rightarrow \mu^{-}+p+X,
$$

where $X$ is a final-state hadronic system consisting of the nuclear remnant with possible additional protons but without pions that indicate RES or other processes. In Eq. (2), the incident neutrino energy $E_{\nu}$ is unknown, but the dependence of $\delta \vec{p}$ on $E_{\nu}$ can be removed. This can be done as follows:
First, decompose $\delta \vec{p}$ into longitudinal and transverse components with respect to the neutrino direction,

$$
\begin{gathered}
\delta \vec{p} \equiv\left(\delta p_{L}, \delta \vec{p}_{T}\right), \\
E_{\nu}=p_{L}^{\mu}+p_{L}^{p}-\delta p_{L}, \\
\overrightarrow{0}=\vec{p}_{T}^{\mu}+\vec{p}_{T}^{p}-\delta \vec{p}_{T},
\end{gathered}
$$

where $\vec{p}^{\mu}$ and $\vec{p}^{p}$ are the muon and proton momenta, respectively. The direction of the transverse momentum imbalance $\delta \vec{p}_{T}$ (see schematic definition in Fig. 1),

$$
\delta \alpha_{T} \equiv \arccos \frac{-\vec{p}_{T}^{\mu} \cdot \delta \vec{p}_{T}}{p_{T}^{\mu} \delta p_{T}}
$$

is uniformly distributed in the absence of IMT because of the isotropic nature of Fermi motion. This variable is thus sensitive to IMT [4]. Because $\left|\vec{p}_{T}^{p}\right|>\left|\vec{p}_{T}^{\mu}\right|$ for $\delta \alpha_{T}<90^{\circ}$, accelerating FSI can be distinguished from decelerating FSI using $\delta \alpha_{T}$. Recent measurements of $\delta p_{T}$ and $\delta \alpha_{T}$ on hydrocarbon at beam energy around $600 \mathrm{MeV}$ by the T2K Collaboration can be found in Ref. [17].

Second, under the assumption that $X$ is just the remnant nucleus, $A^{\prime}$, then $\delta p$ gives the magnitude of its recoil momentum, and

$$
\begin{gathered}
\delta p_{L}=\frac{1}{2} R-\frac{m_{A^{\prime}}^{2}+\delta p_{T}^{2}}{2 R}, \\
R \equiv m_{A}+p_{L}^{\mu}+p_{L}^{p}-E^{\mu}-E^{p},
\end{gathered}
$$

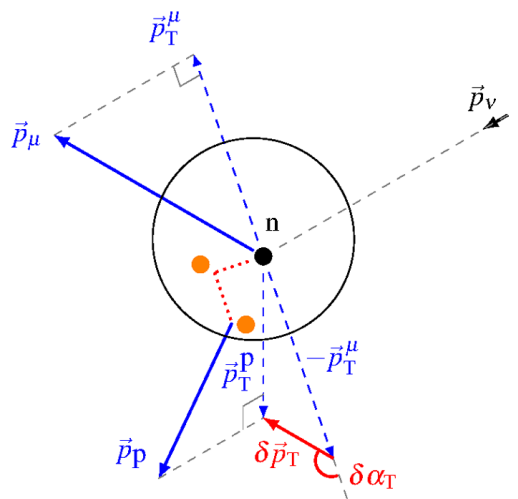

FIG. 1. Schematic definition of the transverse kinematics [4]. 
where $m_{A^{(\prime)}}, E^{\mu(p)}$ are the nuclear target (remnant) mass, and the muon (proton) energy, respectively [5]. In the limit of zero IMT (that is, pure QE), the recoiling momentum of $A^{\prime}$ balances the initial neutron momentum and

$$
p_{n}=\delta p,
$$

which can be estimated using the relation $m_{A^{\prime}}=$ $m_{A}-m_{n}+b$, where $m_{n}$ is the neutron mass, and $b=$ $+27.13 \mathrm{MeV}$ for carbon obtained from the probabilistic model for excitation energy [5].

This Letter presents the measurement of $\delta \alpha_{T}$ and $p_{n}$ in $\nu_{\mu}$ induced production on polystyrene by the MINERvA experiment. The signal is defined as an event with no pions, one muon, and at least one proton satisfying

$$
\begin{array}{cc}
1.5 \mathrm{GeV} / c<p_{\mu}<10 \mathrm{GeV} / c, & \theta_{\mu}<20^{\circ}, \\
0.45 \mathrm{GeV} / c<p_{p}<1.2 \mathrm{GeV} / c, & \theta_{p}<70^{\circ},
\end{array}
$$

where $p_{\mu}$ and $\theta_{\mu}\left(p_{p}\right.$ and $\left.\theta_{p}\right)$ are the muon (proton) momentum and polar angle with respect to the neutrino direction, respectively, when exiting the nucleus. Nuclear effects in terms of Fermi motion and IMT are measured and compared to model predictions.

The MINERvA experiment is in the NuMI beam line [18] at Fermilab. The detector is described in detail elsewhere [19]. The tracker is constructed of hexagonal planes which are approximately perpendicular to the incoming neutrino beam and made from triangular scintillator strips. Scintillator strips in adjacent planes are rotated by $60^{\circ}$ with respect to each other, permitting three-dimensional track reconstruction which is efficient up to $70^{\circ}$ from the detector axis. The scintillator is embedded in polystyrene, containing the carbon target nuclei. The MINOS Near Detector is two meters downstream of the MINERvA detector and serves as a magnetized muon spectrometer [20]. The data used in this analysis correspond to $3.28 \times 10^{20}$ protons on target (POT) delivered from 2010 to 2012; the integrated $\nu_{\mu}$ flux prediction $\left(2.88 \times 10^{-8} / \mathrm{cm}^{2} / \mathrm{POT}\right)$ is from Ref. [21].

Neutrino interactions are simulated with GENIE 2.8.4 [22] in both a nominal form, with and without FSI, and also with a MINERvA "tune," (MNVGENIE-V1). The nuclear initial state is modeled as a relativistic Fermi gas [23]. Quasielastic [24], RES, and deep inelastic scattering (DIS) kinematics are modeled with a dipole axial form factor using $M_{A}^{\mathrm{QE}}=0.99 \mathrm{GeV} / c^{2}$, the Rein-Sehgal model [25], and the 2003 Bodek-Yang model [26], respectively. PYTHIA6 [27] and models based on Koba-Nielsen-Olesen scaling [28] are used to describe hadronization. The $h A$ option of GENIE was used to model FSI [29]. The general performance of the GENIE FSI treatment for pions has been demonstrated in data versus simulation comparisons published by MINERvA [30-34].
MNVGENIE-V1 includes two-particle-two-hole $(2 p 2 h)$ excitations of the nucleus as formulated in the Valencia model [1,35-37]. The interaction strength with $2 p 2 h$ has been tuned to MINERvA inclusive scattering data [38], resulting in a significant enhancement relative to the Valencia model in a restricted region of energy-momentum transfer. MNVGENIE-V1 also includes a modification to the nonresonant pion production as constrained by deuterium data [39], and collective excitations of the nucleus for the QE channel. The latter are approximated as a superposition of one-particle-one-hole $(1 p 1 h)$ excitations and calculated with the random phase approximation [8]. Because the affected events contribute little to the sample, the effects of nonresonant pion production and RPA in this analysis are negligible.

In the nominal GENIE configuration, FSI are further categorized as follows: (1) Noninteracting proton FSIFSI without pion absorption in which the proton propagates as a free particle. (2) Accelerating proton FSI-FSI without pion absorption in which the proton energy increases as a result of FSI. (3) Decelerating proton FSI-FSI without pion absorption in which the proton energy decreases as a result of FSI. (4) Pion FSI-FSI in which a pion is absorbed.

In addition to GENIE, data are also compared to the predictions of NUWRO [40]. The initial state is modeled either as a local Fermi gas or with a spectral function [11]. FSI are treated as intranuclear cascades of hadronic interactions [41] incorporating the Oset model [42], and $2 \mathrm{p} 2 \mathrm{~h}$ excitations are from the Valencia model $[1,35]$.

Events with at least two reconstructed tracks in the MINERvA tracker satisfying Eqs. (10)-(11) are selected. The muon candidate track must match a track in the MINOS Near Detector, necessitating Eq. (10). The two tracks are combined to determine $p_{\mu}$ and $\theta_{\mu}$, with resolutions of $\sim 8 \%$ at $5 \mathrm{GeV} / c$ and $\sim 0.6^{\circ}$, respectively. The proton candidate is distinguished from possible mesons with specific energy loss $(d E / d x)$. Its momentum $p_{p}$ is determined according to the $d E / d x$ profile along the track $[43,44]$. If two or more protons satisfy Eq. (11) (less than $1 \%$ of all selected events), the most energetic one is taken as the proton candidate.

To improve $p_{p}$ resolution, additional selection requirements to obtain elastically scattered and contained (ESC) protons [45] are introduced. When a proton is not contained in the tracker or undergoes inelastic scattering it has a deteriorated momentum estimate. ESC protons are selected by requiring large $d E / d x$ near the track end points. This reduces the spread of the reconstructed $p_{p}$ to about $60 \%$ of its previous measurement [44], resulting in a resolution of $\sim 2 \%$ at $1 \mathrm{GeV} / c$, at the cost of a reduction of statistics to about $40 \%$ of the initial proton sample.

The efficiency (including acceptance effects) of event selection is estimated to be $8.6 \%$ and the purity is $78 \%$. The predicted background contributions mainly come from RES where the pion from baryon decay exits the nucleus 


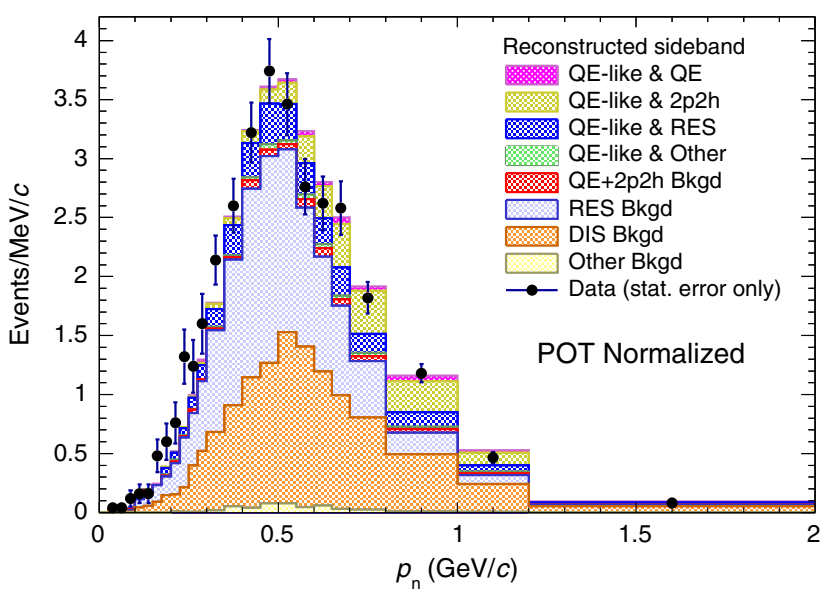

FIG. 2. Reconstructed data distribution of $p_{n}$ in a sideband region, compared to full MNVGENIE-V1+GEANT4 simulation after background tuning. The QE-like signal and the background are decomposed into interaction modes.

but is not identified (13.4\%) and DIS (5.4\%). A data-driven method [44] is used to determine backgrounds. Sidebands are determined in the plane of unattached visible energy away from the interaction vertex vs transferred fourmomentum squared, $Q^{2}$. Backgrounds from the GENIE simulation are rescaled so that the sidebands describe the data (see Fig. 2) and are then extrapolated into the signal region. The background-subtracted distributions are then unfolded [46] with four iterations, where the number of iterations is chosen to balance between the bias and fluctuation of the unfolded distributions. After a subsequent efficiency correction, event distributions are normalized by the product of the number of target nucleons $\left(3.11 \times 10^{30}\right)$, POT, and $\nu_{\mu}$ flux, to obtain the flux-averaged differential cross sections.

Systematic uncertainties are evaluated for all observables. As an example, the cross section uncertainty in $\delta \alpha_{T}$ is summarized here. Besides statistical uncertainty $(5 \%-7 \%)$, uncertainties arising from the NuMI flux prediction $(6 \%)$, GENIE modeling (6\%-9\%), and detector response (6-16\%) are accounted for. For the latter two, parameters in the physics and detector models are varied within uncertainties and the resulting cross section variations are the assigned systematic uncertainties [44]. GENIE model uncertainties predominantly arise from modeling $2 p 2 h$, while the transverse projection of the muon and proton kinematics and the ESC proton selection have significant contributions from detector systematics. The total uncertainty is $20 \%$ at $\delta \alpha_{T}=0^{\circ}$ and $180^{\circ}$, and decreases to about $13 \%$ at $90^{\circ}$.

Muon momentum and polar angle distributions are satisfactorily described by MNVGENIE-V1 (Supplemental Material 1, Fig. 1 [47]), as is the proton momentum distribution (Supplemental Material 1, Fig. 2 [47]). There is a significant overprediction beyond $1-\sigma$ total uncertainty at $\theta_{p} \gtrsim 60^{\circ}$ (Fig. 3). Of the four FSI categories,

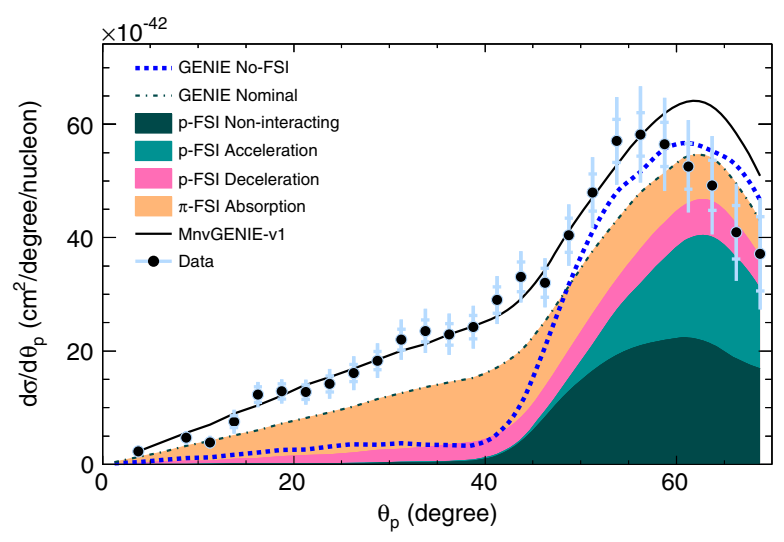

FIG. 3. Differential cross section in $\theta_{p}$ compared to GENIE predictions (nominal GENIE version: 2.8.4). Outer error bars represent the combined statistical and systematic uncertainties, while the inner ones statistical only. The white space between the lines for nominal GENIE and MNVGENIE-V1 is mostly from the tuned $2 p 2 h$ component in the latter.

noninteracting proton and accelerating proton FSI are the ones that appear only in this high angle region. Away from it, at $\theta_{p}$ below $40^{\circ}$ where pion FSI and $2 p 2 h$ contributions are large, the MNVGENIE-V1 prediction describes the data very well.

For GENIE without FSI and nominal GENIE with noninteracting proton, the pure QE assumption of Eqs. (7)-(9) is valid. The corresponding predictions show a Fermi-gas peak at $p_{n} \lesssim 0.25 \mathrm{GeV} / c$ and the additional Bodek-Ritchie tail [23] (Fig. 4). As only Fermi motion is considered for such pure $\mathrm{QE}$ events, the distribution of $\delta \alpha_{T}$ is flat to first order; a secondary effect arises from the cross section dependence on the center-of-mass energy of the interacting neutrino-neutron system (Fig. 5).

With IMT, $\delta p$, and therefore $p_{n}$ [Eq. (9)], is (the modulus of) the convolution of Fermi motion and IMT [Eq. (1)]. As Fermi motion alone has a small effect on $\delta \alpha_{T}$, the effect of

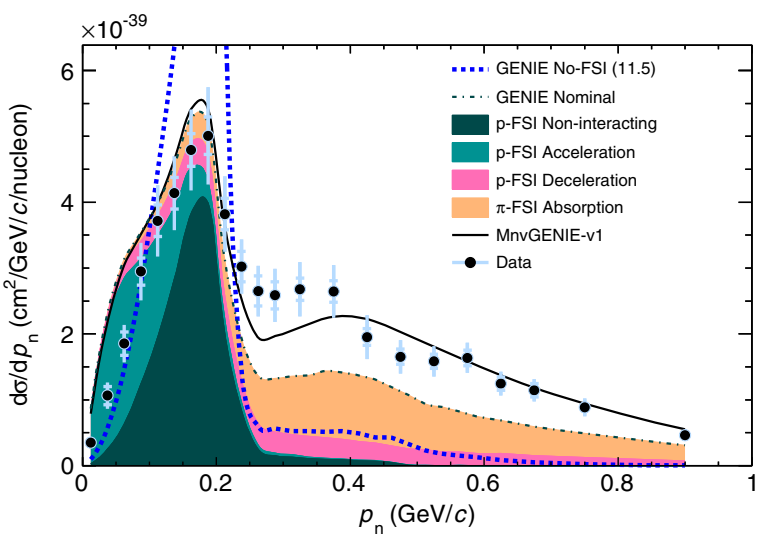

FIG. 4. Differential cross sections in $p_{n}$, compared to GENIE predictions. The no-FSI prediction maximum is 11.5 (see legend) in unit of $10^{-39} \mathrm{~cm}^{2} / \mathrm{GeV} / c /$ nucleon. 


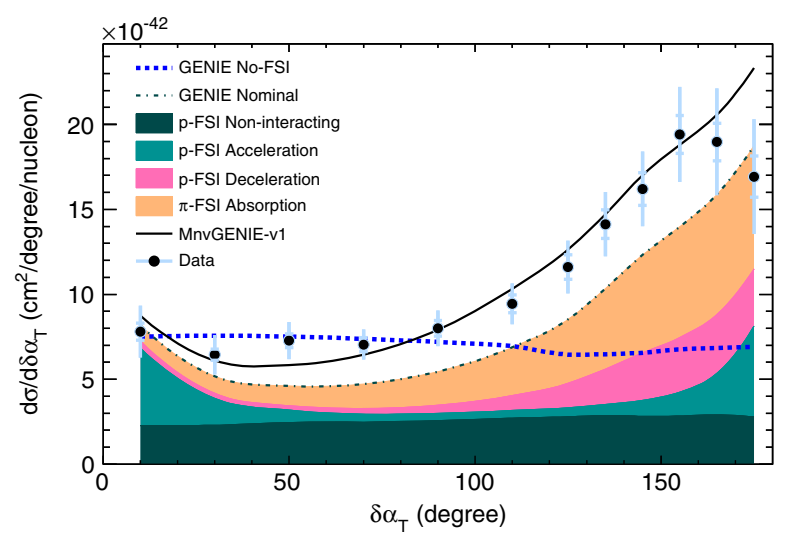

FIG. 5. Differential cross sections in $\delta \alpha_{T}$, compared to GENIE predictions. The peak in accelerating proton FSI on the right side is from events where $\vec{p}_{T}^{p}$ has a large angular separation from $-\vec{p}_{T}^{\mu}$ despite $\left|\vec{p}_{T}^{p}\right|>\left|\vec{p}_{T}^{\mu}\right|$.

IMT is revealed by the nonflatness of the $\delta \alpha_{T}$ distribution. Following the definition Eq. (6) (see also Fig. 1), it can be seen that when $\left|\vec{p}_{T}^{p}\right|<\left|\vec{p}_{T}^{\mu}\right|, \delta \alpha_{T}$ becomes larger than $90^{\circ}$. Since in the plane transverse to the neutrino direction the muon and proton momenta are back to back for a free nucleon target (no nuclear effects), $\left|\vec{p}_{T}^{p}\right|<\left|\vec{p}_{T}^{\mu}\right|$ means that the proton is (transversely) decelerated by nuclear effects. As a result, in Fig. 5, the nominal GENIE prediction with decelerating proton FSI does not contribute greatly to the transverse forward boosting region $\delta \alpha_{T} \lesssim 90^{\circ}$ [4], where accelerating proton FSI are the dominating IMT. Such accelerating FSI are responsible for the QE peak distortion beyond 5- $\sigma$ total uncertainty at the lowest $p_{n}$ (Fig. 4). Of this distortion, the largest contribution comes from the elastic interaction of the GENIE hA FSI model [4] which does not occur in other models, such as GENIE' s hN FSI model [29]. This elastic interaction was designed to resemble hadron-nucleus elastic scattering where the scattered particle could gain energy at the expense of a decelerated recoil nucleus. Turning this effect off can provide a better shape agreement with data.

Nonexclusive IMT-pion FSI and $2 p 2 h$-are well separated from the Fermi motion prediction of the $p_{n}$ QE peak (Fig. 4). At $p_{n}$ below $0.25 \mathrm{GeV} / c$, data points constrain the modeling of Fermi motion; at $p_{n} \gtrsim 0.4 \mathrm{GeV} / c$, where pion FSI and $2 p 2 h$ effects are large, MNVGENIE-V1's prediction follows the data. In the transition region, $p_{n} \sim 0.3 \mathrm{GeV} / c$, MNVGENIE-V1 shows a clear deficit beyond $1-\sigma$ total uncertainty. With pion absorption and $2 p 2 h$ events, the measured proton carries a fraction of the total momentum transfer given to multiple particles. Therefore, these reactions behave similarly as decelerating proton FSI in both $p_{n}$ and $\delta \alpha_{T}$. The overall MNVGENIE-V1 prediction describes the $\delta \alpha_{T}$ distribution well (Fig. 5).

Comparison to the predictions of NUWRO are shown in Fig. 6. Here, NuWro's spectral function model works better than its local Fermi gas model. Like MNVGENIE-V1,
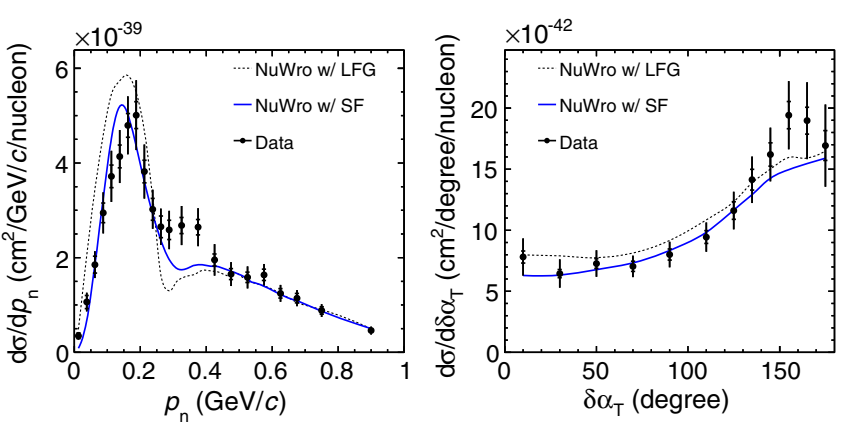

FIG. 6. Differential cross sections in $p_{n}$ (left) and $\delta \alpha_{T}$ (right), compared to NUWRO predictions with local Fermi gas (LFG) or spectral function $(\mathrm{SF})$.

NuWRO with spectral function is lacking strength at the transition region between Fermi motion and non-exclusive IMT (RES and $2 p 2 h$ ), also with a deficit significantly beyond 1- $\sigma$ total uncertainty. Except for this, the pion production and $2 p 2 h$ treatment in NUWRO provide a good description for IMT. Furthermore, the predictions with spectral function for single particle kinematics are all within 1- $\sigma$ total uncertainties (Supplemental Material 1, Figs. 3 and 4 [47]).

NUWRO's spectral function option does introduce a highmomentum component for the initial nucleon motion, more than the Bodek-Ritchie addition gives to GENIE. The spectral function enhancement with respect to local Fermi gas appears in the transition region $p_{n} \sim 0.3 \mathrm{GeV} / c$. This is also a kinematic region where the $2 p 2 h$ tuning process of MNVGENIE-V1 increases the cross section predictions up to $0.4 \times 10^{-39} \mathrm{~cm}^{2} / \mathrm{GeV} / c /$ nucleon. So introduction of such a tuning process to NUWRO with spectral function, or a spectral function-based MNVGENIE-V1 might produce a model that agrees better with the data.

In conclusion, the cross sections of QE-like production in terms of $p_{n}$ and $\delta \alpha_{T}$, whereby the target Fermi motion and the intranuclear momentum transfer are separated, have been presented. MINERvA's tuned implementation of $2 p 2 h$ processes gives rate and shape corrections that enable GENIE to accurately describe the data. Both MNVGENIE-V1 and NUWRO with spectral function provide good descriptions of the single particle kinematics and reasonable predictions for $p_{n}$ and $\delta \alpha_{T}$. However, both generators fail in the transition region between Fermi motion and IMT dominated regions. Combination of a MNVGENIE-V1-like $2 p 2 h$ tune and spectral function might correct this.

This technique relies on the fine-grained tracking capability of MINERvA to identify and precisely measure ESC protons. This technique will also be used in experiments with homogeneous trackers such as liquid argon time projection chambers $[48,49]$.

Because the variables $p_{n}$ and $\delta \alpha_{T}$ have sensitivity to initial- and final-state nuclear effects, their measurements on other nuclear targets from MINERvA and from liquid argon experiments should reveal individual $A$ dependence 
for Fermi motion and IMT such as FSI and $2 p 2 h$, and thus provide a detailed modeling of the nuclear effects that will be valuable for future neutrino oscillation experiments.

This work was supported by the Fermi National Accelerator Laboratory under U.S. Department of Energy contract No. DE-AC02-07CH11359 which included the MINERvA construction project. Construction support was also granted by the United States National Science Foundation under Grant No. PHY-0619727 and by the University of Rochester. Support for participating scientists was provided by NSF and DOE (USA), by CAPES and CNPq (Brazil), by CoNaCyT (Mexico), by Proyecto Basal FB 0821, CONICYT PIA ACT1413, Fondecyt 3170845 and 11130133 (Chile), by Consejo Nacional de Ciencia, Tecnología e Innovación Tecnológica (CONCYTEC), Dirección de Gestión de la Investigación - Pontificia Universidad Católica del Peru (DGI-PUCP), and ViceRectorate for Research of National University of Engineering (VRI-UNI) (Peru), by the Latin American Center for Physics (CLAF), by Science and Technology Facilities Council (UK), and by NCN Opus Grant No. 2016/ 21/B/ST2/01092 (Poland). We thank the MINOS Collaboration for use of its near detector data. We acknowledge the dedicated work of the Fermilab staff responsible for the operation and maintenance of the beam line and detector and the Fermilab Computing Division for support of data processing.

"Present address: Lawrence Berkeley National Laboratory, Berkeley, California 94720, USA.

"Present address: University of Michigan, Ann Arbor, Michigan 48109, USA.

"Present address: University College London, London WC1E 6BT, United Kingdom.

${ }^{\S}$ Present address: Tufts University, Medford, Massachusetts 02155, USA.

[1] J. Nieves, I. Ruiz Simo, and M. J. Vicente Vacas, Phys. Lett. B 707, 72 (2012).

[2] P. Coloma and P. Huber, Phys. Rev. Lett. 111, 221802 (2013).

[3] K. Abe et al. (T2K Collaboration), Phys. Rev. D 96, 092006 (2017).

[4] X.-G. Lu, L. Pickering, S. Dolan, G. Barr, D. Coplowe, Y. Uchida, D. Wark, M. O. Wascko, A. Weber, and T. Yuan, Phys. Rev. C 94, 015503 (2016).

[5] A. P. Furmanski and J. T. Sobczyk, Phys. Rev. C 95, 065501 (2017).

[6] S. K. Singh and E. Oset, Nucl. Phys. A542, 587 (1992).

[7] A. Gil, J. Nieves, and E. Oset, Nucl. Phys. A627, 543 (1997).

[8] J. Nieves, J. E. Amaro, and M. Valverde, Phys. Rev. C 70, 055503 (2004); 72, 019902(E) (2005).

[9] J. Nieves, M. Valverde, and M. J. Vicente Vacas, Phys. Rev. C 73, 025504 (2006).

[10] M. Martini, M. Ericson, G. Chanfray, and J. Marteau, Phys. Rev. C 80, 065501 (2009).
[11] O. Benhar, A. Fabrocini, S. Fantoni, and I. Sick, Nucl. Phys. A579, 493 (1994).

[12] J. Delorme and M. Ericson, Phys. Lett. 156B, 263 (1985).

[13] J. Marteau, J. Delorme, and M. Ericson, Nucl. Instrum. Methods Phys. Res., Sect. A 451, 76 (2000).

[14] M. Martini, M. Ericson, G. Chanfray, and J. Marteau, Phys. Rev. C 81, 045502 (2010).

[15] J. Nieves, I. Ruiz Simo, and M. J. Vicente Vacas, Phys. Rev. C 83, 045501 (2011).

[16] M. Martini, M. Ericson, and G. Chanfray, Phys. Rev. C 84, 055502 (2011).

[17] K. Abe et al. (T2K Collaboration), arXiv:1802.05078.

[18] P. Adamson et al. (MINOS Collaboration), Nucl. Instrum. Methods Phys. Res., Sect. A 806, 279 (2016).

[19] L. Aliaga et al. (MINERvA Collaboration), Nucl. Instrum. Methods Phys. Res., Sect. A 743, 130 (2014).

[20] D. G. Michael et al. (MINOS Collaboration), Nucl. Instrum. Methods Phys. Res., Sect. A 596, 190 (2008).

[21] L. Aliaga et al. (MINERvA Collaboration), Phys. Rev. D 94, 092005 (2016); 95, 039903 (2017).

[22] C. Andreopoulos et al., Nucl. Instrum. Methods Phys. Res., Sect. A 614, 87 (2010).

[23] A. Bodek and J. L. Ritchie, Phys. Rev. D 23, 1070 (1981).

[24] C. H. Llewellyn Smith, Phys. Rep. 3, 261 (1972).

[25] D. Rein and L. M. Sehgal, Ann. Phys. (N.Y.) 133, 79 (1981).

[26] A. Bodek and U. K. Yang, J. Phys. G 29, 1899 (2003).

[27] T. Sjostrand, S. Mrenna, and P. Z. Skands, J. High Energy Phys. 05 (2006) 026.

[28] Z. Koba, H. B. Nielsen, and P. Olesen, Nucl. Phys. B40, 317 (1972).

[29] S. A. Dytman and A. S. Meyer, AIP Conf. Proc. 1405, 213 (2011).

[30] B. Eberly et al. (MINERvA Collaboration), Phys. Rev. D 92, 092008 (2015).

[31] T. Le et al. (MINERvA Collaboration), Phys. Lett. B 749, 130 (2015).

[32] C. L. McGivern et al. (MINERvA Collaboration), Phys. Rev. D 94, 052005 (2016).

[33] O. Altinok et al. (MINERvA Collaboration), Phys. Rev. D 96, 072003 (2017).

[34] M. Betancourt et al. (MINERvA Collaboration), Phys. Rev. Lett. 119, 082001 (2017).

[35] J. T. Sobczyk, Phys. Rev. C 86, 015504 (2012).

[36] R. Gran, J. Nieves, F. Sanchez, and M. J. Vicente Vacas, Phys. Rev. D 88, 113007 (2013).

[37] J. Schwehr, D. Cherdack, and R. Gran, arXiv:1601.02038.

[38] P. A. Rodrigues et al. (MINERvA Collaboration), Phys. Rev. Lett. 116, 071802 (2016).

[39] C. Wilkinson, P. Rodrigues, S. Cartwright, L. Thompson, and K. McFarland, Phys. Rev. D 90, 112017 (2014).

[40] T. Golan, C. Juszczak, and J. T. Sobczyk, Phys. Rev. C 86, 015505 (2012).

[41] V. R. Pandharipande and S. C. Pieper, Phys. Rev. C 45, 791 (1992).

[42] L. L. Salcedo, E. Oset, M. J. Vicente-Vacas, and C. GarciaRecio, Nucl. Phys. A484, 557 (1988).

[43] T. Walton, Hampton University, Report No. FERMILABTHESIS-2014-11. 
[44] T. Walton et al. (MINERvA Collaboration), Phys. Rev. D 91, 071301 (2015).

[45] X.-G. Lu et al. (MINERvA Collaboration), J. Phys. Conf. Ser. 888, 012120 (2017).

[46] G. D’Agostini, Nucl. Instrum. Methods Phys. Res., Sect. A 362, 487 (1995).

[47] See Supplemental Material at http://link.aps.org/ supplemental/10.1103/PhysRevLett.121.022504 for the model-data comparisons for single particle kinematics and $\delta p_{T}$, as well as for the data release including the measured cross sections, their uncertainties and covariance matrices, and the decomposition of the total uncertainties.

[48] M. Antonello et al. (MicroBooNE and LAr1-ND and ICARUS-WA104 Collaborations), arXiv:1503.01520.

[49] R. Acciarri et al. (DUNE Collaboration), arXiv:1512 .06148 . 